\title{
Aiming for operability by habit training in the baseball and all sports
}

\begin{abstract}
Authors and colleagues have been involved with rehabilitation, sports medicine, Masters athlete, pole exercise, baseball and so on. The author Moriyasu was an excellent batter and won the leading hitter prize in the high school. He is now a physiotherapist, sports trainer and emphasizes the operability factor in a sports textbook "You can see in the video! Habit training for baseball skills". Its characteristic points are i) a variety of useful photos, ii) 131 QR codes connecting to videos, iii) observing videos by smartphone anytime, anywhere. This textbook will definitely contribute all people related to baseball and other sports.
\end{abstract}

Volume 5 Issue 2 - 2020

\author{
Akito Moriyasu, ${ }^{1,2}$ Mitsuru Murakami, ${ }^{3}$ \\ Hiroshi Bando, 4,5 \\ 'Rehabilitation Research Group for body and heart in Shikoku, \\ Japan \\ ${ }^{2}$ Akiboshi Bright Star training rehabilitation center, Japan \\ 3apan Masters Athletics, Kagawa division, Japan \\ ${ }^{4}$ Tokushima University, Medical Research, Japan \\ 5Japan Masters Athletics, Tokushima division, Japan
}

Correspondence: Hiroshi Bando, Medical Research,
Tokushima University,Nakashowa I-6I,Tokushima 770-0943,
Japan,Tel+8I-90-3187-2485, Email pianomed@bronze.ocn.ne.jp

Received: April 0I, 2020 | Published: April 13, 2020

\section{Introduction}

Authors and colleagues have been involved in anti-aging medicine, medical care, rehabilitation, sports medicine, Masters athletics, baseball and fitness. ${ }^{1}$ Especially, the method of pole exercise has been beneficial to treat healthy people and patients with various diseases and impairments. ${ }^{2}$ We provide health advice for people with lifestyle-related diseases on diet and exercise, and provide medical rehabilitation guidance for those with orthopedic diseases. ${ }^{3}$ Furthermore, we have continued a variety of approaches to sports enthusiasts and professional athletes of all ages, as well as Masters athletes, para-athletes aiming for the Para-Olympics. ${ }^{4}$ Our projects include international English medical papers and presenting lectures and workshops at international and domestic academic conferences. ${ }^{5}$

Under such circumstances, there have been various experiences between the authors and baseball for years. ${ }^{6}$ The first author Moriyasu was an excellent batter in the high school baseball team. He won the leading hitter prize with high batting average, which was like Ichiro in the Major League Baseball (MLB). After that, he became a physiotherapist, sports trainer and director of sports rehabilitation association. Thanks to his persistant coaching, Sambonmatsu high school could participate all Japan baseball tournament in Koshien. ${ }^{6} \mathrm{He}$ has been teaching and supporting many professional soccer and baseball players as sports trainers.

The second author Murakami ran fast 10.9 seconds for $100 \mathrm{~m}$ during university student. After that, he has taught many athletes as school teacher and masters' lecturer. He had a new record at $200 \mathrm{~m}$ dash on Masters Athletic games. ${ }^{7}$ Recently, he has given many lectures and workshops how to run fast without injury with his written textbook for many athletes and high school baseball students.

The third author Bando had been a soft-ball pitcher and baseball player for more than half century. After graduation of university, he turned to a switch hitter, then he won the leading hitter prize a few times in the baseball league. He can run 13.0 seconds for $100 \mathrm{~m}$ dash for half century, and had a new record at $60 \mathrm{~m}$ and $100 \mathrm{~m}$ dash on Masters Athletic games. ${ }^{7}$
Based on the background described above, we have recently published a new book (Figure 1). ${ }^{6}$ The title is "You can see in the video! Habit training for baseball skills". The subjects for this book include junior and senior high school and college baseball players, and their coaches, parents, and related people, as well as all other athletes and their stakeholders. Its content covers broad areas, such as physical function to be checked, habit training to prevent injuries and improve performance, smooth movement without twisting, flat grounding, and so on. These contents were explained in detail from various aspects. ${ }^{6}$

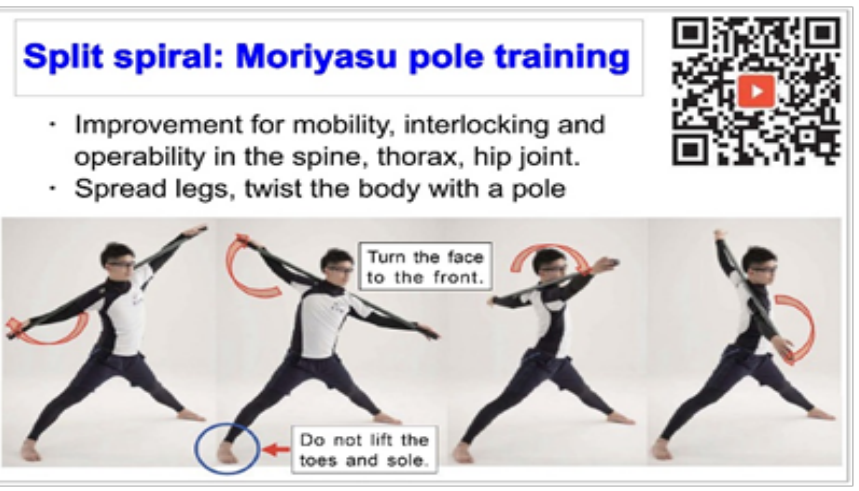

Figure I Sports textbook "You can see in the video! Habit training for baseball skills".

The characteristic points of this book are in the following: i) a variety of useful photos are included for everyone to understand the training well, ii) there are $131 \mathrm{QR}$ codes which can instantly connect to motion videos showing how to perform each fitness, iii) anyone can train oneself anytime, anywhere by observing the motion pictures with a smartphone. ${ }^{6}$ Consequently, everybody can read the explanations, understand well with video and practice any kind of fitness accompanied with smartphone. This textbook will definitely contribute all people who are related to baseball and other sports.

One example training from the book is shown in Figure $2 .^{6}$ This scene is the operation practice of split spiral using the pole exercise. 
The important tip is to acquire mobility, interlocking and operability in the series of spine, thorax and hip joint. ${ }^{8}$ In particular, operability is indispensable for various movements in the baseball. ${ }^{9}$ Regardless of the pitching or batting, the power starts from the lower body and waist, which is transmitted from the thorax to the shoulders, upper arms and forearms. ${ }^{10}$ These flexible movements are crucial without excessive muscle tension. ${ }^{11}$ Consequently, we hope that adequate operability will be acquired for various movements in the baseball and other sports associated with maintaining coordination.

In summary, a training textbook for baseball and other sports was introduced in this article. It is useful for learning mobility, interlocking and operability with QR codes observing exercise videos. Especially, the operability is crucial factor for baseball. We hope that this paper will be useful to athletes and stakeholders of various disciplines.

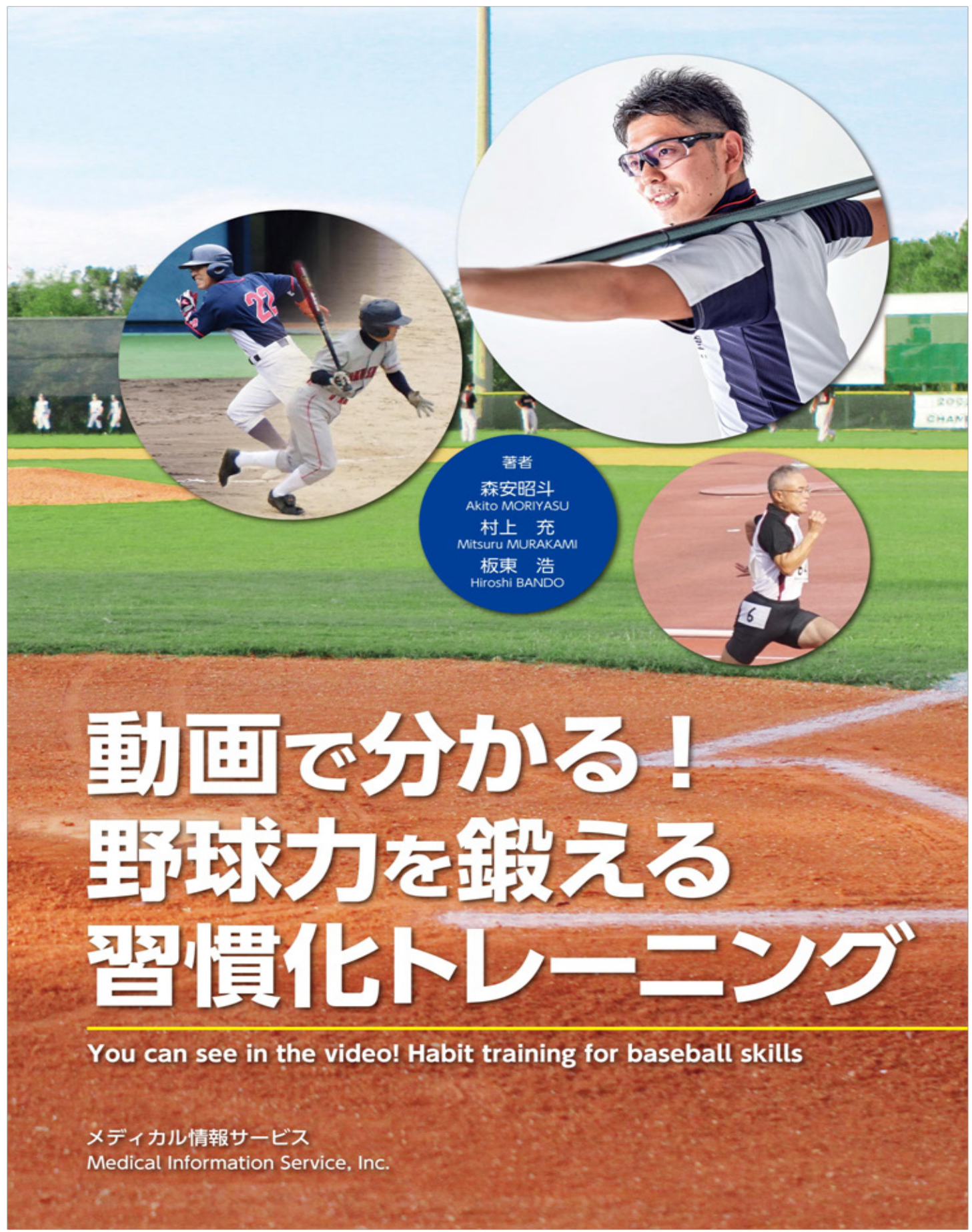

Figure 2 One of the exercise "Split spiral: Moriyasu pole training". 


\section{Acknowledgments}

Authors express our gratitude for related people concerning this study.

\section{Conflicts of interest}

The authors declare no conflict of interest.

\section{Referencs}

1. Bando H, Moriyasu A, Murakami M. Recent trends of common factors of geriatci medicine and rehabilitation medicine. $J$ Helath Med Res. 2020;2(1):1-3.

2. Kurihara R, Fujimoto D, Dakashita T, et al. The influence of Pole exercise on the range of motion of thoracic spine. Clinical Research in Orthopaedics. 2019;2(1):1-5.

3. Moriyasu A, Bando H, Murakami M. Practically useful pole exercise in rehabilitation for improved rib cage flexibility. Prog Rehab Scie J. 2019;1(1):1-3.

4. Murakami M, Bando H. Smooth running without power on sole hallucal area. Res Inves Sports Med. 2018;3(5).

5. Bando H, Yoshioka A, Nishikiori Y. Various care option of integrative medicine from the viewpoint of patient-oriented medicine. Int J Conf Proc. 2020;2(1).
6. Moriyasu A, Murakami M, Bando H. You can see in the video! Habit training for baseball skills. Medical Information Service, Co., Ltd. Tokyo, Japan. 2020: ISBN 978-4-903906-18-8.

7. Bando H, Murakami M, Moriyasu A. Beneficial flat grounding for sprint running by the mechanism of forward leaning and bending knees. Acta Scientific Orthopaedics. 2020:3.4.

8. Sasaki K, Hongo M, Miyakoshi N, et al. Evaluation of sagittal spinepelvis-lower limb alignment in elderly women with pelvic retroversion while standing and walking using a three-dimensional musculoskeletal model. Asian spine journal. 2017;11(4):562-569.

9. Hey HWD, Tan KA, Chin BZ, et al. Comparison of whole body sagittal alignment during directed versus natural, relaxed standing postures in young, healthy adults. The Spine Journal. 2019;19(11):1832-1839.

10. Bernstein J, Charette R Sloan M, et al. Spinal fusion is associated with changes in acetabular orientation and reductions in pelvic mobility. Clin Orthopaed Relat Res. 2019;477(2):324-330.

11. Murakami M, Bando H, Moriyasu A. Flexibility of the chest-lumbar region in athletic athletes. Int Phys Med Rehab J. 2019;4(5):207-208. 